\title{
HÁBITAT URBANO EN LA SEGUNDA DÉCADA DEL SIGLO XXI: EXPERIENCIA CUBANA
}

\author{
HABITAT URBANO NA SEGUNDA DÉCADA DO SÉCULO XXI: EXPERIÊNCIA \\ CUBANA
}

URBAN HABITAT IN THE SECOND DECADE OF THE $27^{\text {st }}$ CENTURY: CUBAN EXPERIENCE

\author{
Dania González Couret ${ }^{7}$ (i) \\ Universidad Tecnológica de La Haban, Cuba
}

Resumen: Como alternativa al modelo hegemónico en la región, Cuba aboga por mantener una sociedad inclusiva y justa con la mejor calidad de vida posible para todos, en un difícil contexto político. El artículo pretende una reflexión crítica sobre aportes y limitaciones en realizaciones teóricas y prácticas de la última década, resultado del trabajo continuado de un grupo de investigación que articula ciencia, formación y extensión como respuesta a problemas, objetivos y proyectos, en la construcción de un cuerpo teórico que sirve de base metodológica a la evaluación y propuesta de soluciones innovadoras. Partiendo del marco teórico y los antecedentes desde la segunda mitad del siglo $X X$, se presentan y discuten algunas experiencias y retos futuros. Se destacan las fortalezas del modelo cubano como el sentido de comunidad, solidaridad, organización social, resiliencia, iniciativa, creatividad, desarrollo humano, capital científico, y la presencia de un Estado que vela por la justicia y equidad. Los retos fundamentales se refieren a la urgente aprobación de la política y la ley que guíe el desarrollo del hábitat urbano bajo la gestión integrada de una institución única, un mejor aprovechamiento del capital científico técnico en ese campo y evitar la estratificación de la ciudad.

Palabras clave: Hábitat urbano; Siglo XXI; Cuba

Resumo: Como alternativa ao modelo hegemônico na região, Cuba defende a manutenção de uma sociedade inclusiva e justa com a melhor qualidade de vida possível para todos, em um contexto político difícil. $\bigcirc$ artigo busca uma reflexão crítica sobre as contribuições e limitações das conquistas teóricas e práticas da última década, fruto do trabalho contínuo

\footnotetext{
1 Arquitecta, 1979, Doctora en Ciencias Técnicas (PhD.), 1994; Doctora en Ciencias, 2007. Profesora Titular (2001), Universidad Tecnológica de La Habana (CUJAE); Miembro Academia de Ciencias de Cuba (2012). E-mail:daniagcouret@gmail.com
} 
de um grupo de pesquisa que articula ciência, formação e extensão como resposta a problemas, objectivos e projectos, na construção de um corpo teórico que serve de base metodológica para a avaliação e proposta de soluções inovadoras. Partindo do referencial teórico e dos antecedentes da segunda metade do século $X X$, experiências e desafios futuros são apresentados e discutidos. Destacam-se os pontos fortes do modelo cubano, como sentido de comunidade, solidariedade, organização social, resiliência, iniciativa, criatividade, desenvolvimento humano, capital científico e a presença de um Estado que zela pela justiça e equidade. Os desafios fundamentais referem-se à aprovação urgente da política e da Lei que orienta o desenvolvimento do habitat urbano sob a gestão integrada de uma única instituição, um melhor aproveitamento do capital técnico-científico nesta área e evitando a estratificação da cidade.

Palavras-chave: Habitat urbano; Século XXI; Cuba

Abstract: As an alternative to the hegemonic model in the region, Cuba advocates maintaining and inclusive and just society with the best possible quality of life for all, in a difficult political context. The article seeks a critical reflection on contributions and limitations in the theoretical and practical achievements of the last decade, as a result of the continuous work of a research group that articulates science, training and extension in response to problems, objectives and projects, in the construction of a theoretical body that serves as a methodological basis for the evaluation and proposal of innovative solutions. Starting from the theoretical framework and the antecedents from the second half of the $20^{\text {th }}$ century, some experiences and future challenges are presented and discussed. The strengths of the Cuban model stand out, such as the sense of community, solidarity, social organization, resilience, initiative, creativity, human development, scientific capital, and the presence of a state that watches over justice and equity. The fundamental challenges refer to the urgent approval of the policy and the law that guides the development of the urban habitat under the integrated management of a single institution, a better use of technical scientific capital in this field and avoiding the stratification of the city.

Keywords: Urban habitat; XXI Century; Cuba 


\section{Introducción}

Cuba representa una alternativa al modelo hegemónico en la región, abogando por mantener una sociedad inclusiva y justa, con la mejor calidad de vida posible para todos, donde el acceso a la vivienda y a la ciudad no estén determinados por las diferencias económicas, sociales, raciales o étnicas. Pero cumplir este objetivo se hace cada vez más difícil en medio de un contexto político, económico, comercial y financiero hostil, signado por el bloqueo impuesto por los Estados Unidos de norteamérica durante más de 60 años, y agravado por los recientes cambios de la correlación de fuerzas ocurridos en la región latinoamericana, en detrimento de los gobiernos progresistas de izquierda. Correlación que ha comenzado a revertirse.

En este marco, se presentan algunas de las principales realizaciones teóricas y prácticas en el campo del hábitat urbano durante los últimos diez años en Cuba, a partir de una reflexión crítica sobre sus aportes y limitaciones en relación con las circunstancias condicionantes, así como los retos futuros. El abordaje integral del tema requiere de la incursión en diversos campos, cada uno de los cuales podría ser tratado por separado de forma detallada, pero en esta ocasión se integran en un enfoque general y crítico del hábitat urbano.

\section{Materiales y métodos}

Los resultados que se presentan corresponden a una época muy reciente, que es la última década, es decir, la segunda del Siglo XXI (2010 - 2020), algunos de los cuales no han sido aún publicados. No se trata, por tanto, de una investigación histórica, sino de la valoración crítica de experiencias locales contemporáneas, en relación con el marco teórico general y las circunstancias condicionantes específicas. Para ello se elabora un marco 
referencial internacional que caracterizará los antecedentes y las tendencias actuales, a partir de la discusión teórica de la documentación consultada y de la experiencia vivida hasta 2020, lo que incluye la pandemia de Covid-19.

Las circunstancias condicionantes, específicas del caso cubano, se caracterizan como resultado de la investigación histórica de los antecedentes desde 1959 y la valoración crítica de la situación actual, sobre la base de la búsqueda documental y el trabajo empírico en el campo del hábitat, en el cual la autora se ha visto directamente involucrada durante las últimas cinco décadas.

La caracterización y valoración crítica de las realizaciones teórico prácticas de las últimas décadas en el país incluyen dos tipos de investigación. Una, fundamentalmente empírica, que ha permitido, en unos casos, describir experiencias prácticas en desarrollo a partir de la consulta a la escasa información publicada, y reflexionar sobre las propias vivencias de la autora, tomando como base investigaciones teóricas previas, algunas de las cuales se exponen en el marco teórico. Otra, propositiva, que recopila propuestas teóricas y metodológicas desarrolladas desde la academia en respuesta a los problemas diagnosticados, con diversos grados de aplicación, en cuya elaboración la autora ha estado involucrada.

Las investigaciones teóricas, empíricas y propositivas cuyos resultados se recogen en el presente artículo han sido dirigidas por la autora en el Grupo de Investigación en Vivienda, el Programa de Maestría en Vivienda Social y el Programa de Doctorado en Arquitectura de la Universidad Tecnológica de La Habana "José Antonio Echeverría" (CUJAE), durante los últimos 20 años.

Sobre esta base, en el artículo se parte de una breve caracterización del marco teórico y los antecedentes que permiten valorar las circunstancias condicionantes, a escala global y local, para luego presentar 
una selección de experiencias prácticas y de propuestas académicas desarrolladas en Cuba durante la última década, cuya discusión posterior permite fundamentar sus fortalezas y debilidades, así como los principales retos futuros a enfrentar.

\section{Resultados}

\subsection{Marco teórico. Antecedentes}

La segunda mitad del siglo XX estuvo marcada por el proceso de urbanización que generó un desproporcionado crecimiento exponencial de las principales ciudades latinoamericanas, agravado por el hecho de que, de manera general, no ha sido planificado, sino producto de la espontaneidad que ha caracterizado la producción social del hábitat (ORTIZ, 1998), cuyo resultado es la ciudad informal, mayoritaria hoy en la región (CEPAL, 2019).

Este fenómeno ya había sido denunciado en la Conferencia Cumbre del Hábitat, celebrada en Vancouver en 1976 (HÁBITAT, 1976), pero entonces se pensaba que el proceso de urbanización podría detenerse o, al menos, atenuarse, elevando la calidad de vida de la población rural. No obstante, las apreciaciones de John F. Turner (1976) en esa década influyeron en el cambio de visión con respecto a la ciudad informal que se estaba gestando, y que dejó de ser considerada como un problema para transformarse en una oportunidad. Esto resultó de gran utilidad a las tendencias neoliberales para transitar del concepto de "vivienda social" gestionada y subsidiada por el "estado de bienestar" hacia el de "vivienda de interés social", donde el Estado se transforma en un simple facilitador del proceso de producción social del hábitat, en el cual intervienen numerosos actores, incluido el sector privado.

En la Conferencia Cumbre Hábitat II, celebrada veinte años después en Estambul (THE HABITAT AGENDA, 1996), se reconoció que el proceso de urbanización era inevitable e irreversible, razón por la cual sería necesario el 
desarrollo de asentamientos humanos sustentables en un mundo en urbanización, siendo luego reconocido, no sólo el derecho a la vivienda (RED-HABITAT, 2008), sino también a la ciudad (RED-HABITAT, 2009). Sin embargo, la urbanización informal, no planificada, generada de forma progresiva por el proceso de producción social del hábitat, si bien es un resultado legítimo y una respuesta popular al fracaso del modelo urbano moderno, nacido con el concepto de la vivienda social masiva, mantiene la segregación urbana de la "ciudad dual" (TEZANOS, 1999) que genera inseguridad y violencia. En ella se pierde el "urbanismo de comunicación" y no resulta sustentable, ya que cualquier proceso de este tipo debe ser planeado de forma integral y participativa, y dirigido a un fin. Por tanto, el derecho a la ciudad no debe asociarse con el de su producción informal.

A partir del reconocimiento de las ventajas de la ciudad histórica con respecto a los nuevos asentamientos periféricos y la aplicación de otros principios de la urbanización moderna como el "zoning" y la separación del vehículo y el peatón, desde la década de los años 80 ganó espacio a escala global (y a partir de los 90 en la región) el reconocimiento de las bondades de la urbanización tradicional y, con ello, el interés por la conservación del patrimonio y el rescate de los centros históricos (ROJAS, 2012) que, de manera general, habían sufrido un proceso de abandono y tugurización, por lo cual predominaba en ellos la inseguridad y la violencia.

Pero las operaciones de rescate de los centros históricos, así como los frentes de agua o las antiguas zonas industriales, mediante operaciones inmobiliarias, supuestamente para el disfrute de todos los ciudadanos, han ocasionado, en realidad, el desplazamiento de los habitantes que las habían ido ocupando durante las últimas décadas de abandono y deterioro, y procesos de gentrificación, como resultado del interés por recuperar las plusvalías del suelo urbano (NAVARRETE, 2017).

\footnotetext{
${ }^{2}$ Según el arquitecto cubano Ricardo Porro, se refiere al que permite la interacción social y humana al deambular por el espacio urbano
} 
Los Objetivos de Desarrollo Sostenible aprobados en 2015 como reemplazo a los incumplidos Objetivos del Milenio, y la Nueva Agenda Urbana resultante de la Conferencia Cumbre Hábitat III celebrada en Quito en 2016 (HIC-AL, 2016) abogan por el desarrollo de ciudades inclusivas, seguras, resilientes y sostenibles, para lo cual es necesario aprender del pasado y de la naturaleza, en aras de promover soluciones diversas, específicas, participativas y de calidad donde se mezclen variados usos del suelo y estratos sociales en pequeñas unidades autónomas interconectadas que prioricen la movilidad peatonal, aprovechando al máximo los recursos disponibles, preferiblemente reusables, reciclados, reciclables y renovables, a la vez que se minimicen los impactos ambientales. Un importante recurso, prácticamente no renovable, a ser aprovechado es el suelo, para evitar la extensión de la mancha urbana con sus consecuencias negativas, además, en transporte e infraestructura, por lo cual, desde hace varias décadas ha habido consenso en cuanto a que la ciudad sustentable ha de ser compacta (BJUR; GAVATIN, 1997; ORRSKOG , 2002; PORTNEY, 2013)

Para ello se requiere de un adecuado ordenamiento jurídico, financiero y físico, así como de políticas y estrategias integrales, sobre la base de una participación verdadera, que propenda hacia ciudades realmente inteligentes en todos los aspectos de la vida, a lo cual, las tecnologías de la información y la comunicación pueden contribuir, si se usan correctamente.

Por último, la pandemia que sufre el planeta desde finales de 2019 ha puesto aún más al descubierto las consecuencias de la fragmentación urbana, y constituye uno de los principales retos futuros para el hábitat urbano, que ya se debate ampliamente hoy en los medios digitales (ALONSO, 2020; MACHORRO, 2020; GUDAY, 2020). La deficiente habitabilidad en la vivienda de la población de menos recursos dificulta "quedarse en casa" y mantener el aislamiento físico, desarrollando, además 
en ella, todas las funciones urbanas que habían sido identificadas y clasificadas por La Carta de Atenas (habitar, trabajar y recrearse). Gran parte de esa población trabaja en el sector informal, por lo cual necesita salir de casa para buscar el sustento de cada día. Aun en los casos en que el teletrabajo fuese posible, cuántas computadoras y conexiones se necesitarían para que los adultos trabajen a la vez que los menores realicen sus labores de aprendizaje, pero, además, cuánto espacio y privacidad requerirían para que todas estas actividades simultáneas no se entorpezcan mutuamente. Por tanto, es evidente que esta "primera epidemia" del siglo XXI influirá en la transformación de las ciudades, como tantas otras antes (BENÉVOLO, 1975), fundamentalmente, en el hábitat de la población de menos recursos, tanto a escala urbana como arquitectónica.

\subsection{Circunstancias condicionantes en Cuba}

Desde el 1ro de enero de 1959 se inició en Cuba un importante proceso de transformaciones políticas, económicas y sociales, cuyo carácter "socialista" fue declarado en abril de 1961, una vez que ya habían sido promulgadas numerosas leyes que apuntaban en esa dirección, como la reforma agraria, la nacionalización de las empresas norteamericanas y nacionales, así como la enseñanza, y la reforma urbana. En materia de vivienda, desde enero de 1959 se prohibieron los desahucios y se creó el Instituto Nacional de Ahorro y Vivienda (INAV), como antecedente de la Dirección de Viviendas Urbanas del Ministerio de Obras Urbanas (1961). En el propio año 1959 se rebajó en un 50\% el valor de los alquileres y en 1960 se promulgó la Ley de Reforma Urbana, mediante la cual se prohibió el arriendo de viviendas y los inquilinos se transformaron en propietarios (SEGRE, 1985).

Entre los ideales de justicia y equidad promovidos desde la década de los 60's se encontraba el afán de reducir las diferencias entre el trabajo manual e intelectual y entre el campo y la ciudad, lo cual se manifestó en el 
intercambio de fuertes flujos de población urbana que iba a trabajar al campo, mientras que los hijos de campesinos comenzaban a estudiar en las ciudades. Se estructuró el sistema nacional de asentamientos humanos (FRANCO PARELLADA, 1989), contribuyendo a reducir la migración campo - ciudad, y se priorizó el desarrollo de las zonas rurales (SEGRE, 1989), gracias a lo cual los centros urbanos históricos no fueron transformados y han podido ser conservados durante las últimas décadas (RIGOL, 2012).

Las oportunidades de estudio que se abrieron para todos generaron escasez de fuerza laboral en sectores como la agricultura y la construcción. Esto, unido a la demanda masiva de vivienda, condicionaron la certeza sobre la necesaria industrialización de la construcción, proceso que se inició en los 60's con la búsqueda de soluciones tecnológicas apropiadas a la economía de un país en desarrollo (VEJAR, 1994), continuando con la adopción de sistemas foráneos de alta tecnología a partir de la década del 70, que tuvieron que ser abandonados, por insustentables, durante la crisis económica de los 90's, momento en el que se transitó hacia el empleo de tecnologías alternativas en el llamado "movimiento de viviendas de bajo consumo material y energético" ${ }^{3}$, pero sin un adecuado conocimiento y transferencia.

Otra forma de contrarrestar la escasez de mano de obra en el sector de la construcción fue la creación de "microbrigadas", organizadas por centros laborales para la construcción de viviendas, que una vez terminadas eran asignadas por la asamblea de trabajadores a aquellos con más necesidad y méritos. Durante la primera etapa de esta experiencia, en la década del 70, se desarrollaron nuevas áreas residenciales urbanas periféricas a partir de proyectos repetitivos (GONZÁLEZ COURET, 2021), mientras que en la segunda mitad de los años 80's, se construyeron edificios en lotes disponibles dentro de la trama urbana consolidada de la

\footnotetext{
3 Para poder continuar construyendo viviendas durante la crisis se adoptó la tecnología del bloque machihembrado de suelo-cemento con techos abovedados y se establecieron indicadores de consumo. Pero esa tecnología, inapropiada al clima cubano, era prácticamente desconocida, y no se transfirió adecuadamente, lo cual afectó la durabilidad de las viviendas ejecutadas.
} 
ciudad (GONZÁLEZ COURET, 2019), como resultado, entre otras razones, de la toma de conciencia sobre el valor de la ciudad histórica.

La inclusión de La Habana Vieja y de su sistema de fortificaciones en la Lista del Patrimonio Mundial en 1982, unida a la tendencia internacional dirigida al reconocimiento de la importancia de los centros históricos, sirvieron de base para el inicio de una importante labor de conservación del patrimonio urbano y arquitectónico en Cuba, cuyo desarrollo durante la última década constituye una de las experiencias a abordar en el presente artículo.

Debido al auge y los resultados demostrados en la autoconstrucción de viviendas por "esfuerzo propio de la población"4 (MICONS, 1989), la Ley General de la Vivienda (48/84) aprobada en 1984 abogó por la promoción de esta modalidad, en paralelo con la gestión estatal, autorizando, además, el alquiler y la compra - venta de viviendas, en aras de favorecer un mejor aprovechamiento del potencial habitacional disponible. Sin embargo, esta ley fue modificada en 1988 (65/88), limitando las posibilidades de renta, que fueron nuevamente autorizadas en $2010^{5}$, y de compraventa, admitidas con posterioridad en $2012^{6}$, mediante documentos modificativos de la Ley 65/88. No obstante, aún hoy, sólo es posible comprar y vender a precio de oferta y demanda en el mercado, viviendas "de segunda mano", ya que no se construyen nuevos edificios destinado a este fin (ONU-HÁBITAT, 2014), pues el negocio inmobiliario iniciado en los años 90's como una vía de salida a la crisis económica conocida como "período especial", decayó en poco tiempo, una vez que su destino fue limitado sólo al alquiler.

\footnotetext{
${ }^{4}$ El término "esfuerzo propio" es el que se usa en Cuba para referirse a la autoconstrucción de viviendas.

${ }^{5}$ Ver Reglamento sobre arrendamiento de viviendas, habitaciones y espacios, publicado en la Gaceta Oficial de Cuba el 8 de octubre de 2010.

${ }^{6}$ Ver Decreto Ley No 288 de 2012.

${ }^{7}$ El "período especial" en tiempo de guerra concebía la posibilidad de supervivencia en caso de guerra y bloqueo total a la isla, pero esta condición se presentó en época de paz, a partir de la caída del muro de Berlín, que interrumpió el intercambio comercial con los países de Europa del Este, lo cual se sumó al bloqueo impuesto por los Estados Unidos desde inicios de los 60's.
} 
A pesar de la llamada "ofensiva revolucionaria" que en 1968 terminó con el pequeño negocio privado, el "trabajo por cuenta propia" se ha mantenido siempre, sobre todo en el sector de los servicios, como, por ejemplo, costura, barbería, peluquería, reparaciones menores, entre otros. Sin embargo, esta modalidad se ha visto impulsada o limitada, según los cambios operados en las condiciones económicas del país ${ }^{9}$. Lo cierto es que los que laboran en este sector, por lo general, perciben mayores ingresos que los trabajadores estatales ${ }^{10}$ a pesar de sus deberes fiscales, pero la mayoría de los profesionales graduados de nivel superior solo pueden ejercer su labor especializada en entidades estatales. Tal es el caso de arquitectos, ingenieros $u$ otros profesionales que tributan a la construcción y el hábitat urbano.

Los cambios de enfoque asumidos en diferentes momentos con respecto a la autoconstrucción, el alquiler y la compra - venta de vivienda, así como al rol del sector no estatal en la esfera productiva y de servicios, responden a la contradicción que estas variables generan entre la necesidad de eliminar algunas de las trabas que limitan la solución del problema, y el afán de evitar el incremento de la estratificación de una sociedad que ha intentado conservar el mayor grado posible de justicia y equidad. Así, la autoconstrucción fue nuevamente impulsada a mediados de la primera década del Siglo XXI, como principal vía para la solución del problema habitacional en Cuba, apoyada por el Programa de Arquitectos de la Comunidad que había sido creado en los 90's. Sin embargo, esa fórmula, a escala familiar aislada, fomenta la extensión de la mancha urbana, con todos los inconvenientes que eso acarrea y no permite el crecimiento en altura, por lo cual no es aplicable en centros de ciudad (GELABERT, 2014). La apuesta por opciones intermedias que eviten estas

\footnotetext{
${ }^{8}$ Término empleado en Cuba para referirse al sector no estatal, ya sea un trabajador individual o un pequeño empresario para el que este trabaje.

${ }^{9}$ Ver Ley 356 de 2018 del Consejo de Estado.

10 Otros sectores de la población también perciben mayores ingresos que los trabajadores estatales, como los beneficiados con las propinas en el turismo; los que laboran en empresas mixtas o extranjeras que son estimulados en adición al salario estatal; los que reciben remesas familiares del exterior, e incluso, dentro del propio sector estatal, se diferencian el deporte y la cultura, y más recientemente, el sistema empresarial en perfeccionamiento.
} 
limitaciones es aún demasiado lenta, y se abordará en los resultados de la última década que serán presentados y discutidos en el presente artículo.

La experiencia de la pasada década (2010 - 2019), en la que se inscriben las realizaciones teórico-prácticas que serán discutidas, está signada por la aprobación de documentos rectores que trazan las pautas de hacia dónde se pretende encaminar el desarrollo del país en el futuro mediato. En orden cronológico pueden citarse los "Lineamientos de la Política Económica y Social del Partido y la Revolución", aprobados en el VI Congreso del Partido Comunista de Cuba (PCC) en abril de 2011, y actualizados para VII Congreso del PCC en 2016, conjuntamente con la "Conceptualización del Modelo Económico y Social Cubano de Desarrollo Socialista", y las bases del "Plan Nacional de Desarrollo Económico y Social hasta el 2030: Visión de la Nación, Ejes y Sectores Estratégicos"111, a los que se suma la nueva Constitución de la República, sometida a referendo aprobatorio en abril de 2019.

De los Lineamientos aprobados en 2011 se derivaron numerosas políticas y sus respectivas normas jurídicas, que han ido apareciendo paulatinamente, encaminadas a actualizar, ordenar y articular un marco legal que había ido surgiendo y siendo modificado sobre la marcha, a partir de la retroalimentación obtenida de su implementación práctica. Particular importancia revisten aquellas encaminadas a fortalecer el potencial científico y tecnológico del país en aras de mejorar la economía y el bienestar de la población, como las dirigidas a la creación de Parques Científico Tecnológicos y Empresas de Alta Tecnología (DÍAZ-CANEL BERMÚDEZ; NÚÑEZ, 2020). Sin embargo, la política y la ley de la vivienda aun hoy, 10 años después, no han sido aprobadas, aunque en ciertas ocasiones se ha pretendido asumir como tal un programa de construcción, propuesto sobre la base de una visión muy parcializada de ese sector, que es quien dirige esta esfera de la vida nacional. "Documentos del 7mo. Congreso del Partido aprobados por el III Pleno del Comité Central del PCC el 18 de mayo
de 2017 y respaldados por la Asamblea Nacional del Poder Popular el 1 de junio de 2017. (DOCUMENTOS, 2017). 


\subsection{Realizaciones teórico - prácticas de la última década}

Las circunstancias condicionantes del hábitat urbano en Cuba durante la segunda década del siglo XXI se caracterizan por la ausencia de un único órgano rector del hábitat, así como de políticas o leyes que orienten y regulen el desarrollo habitacional y urbano. No obstante, se presentarán algunas experiencias, tanto prácticas como académicas, que dan cuenta de enfoques que pueden constituir, si bien no alternativas terminadas y probadas, al menos, puntos de vista divergentes con respecto al modelo predominante en la región.

\subsubsection{La Nueva Agenda Urbana Cubana (NAUC)}

El Instituto de Planificación Física (IPF), que representa a Cuba ante las instancias de ONU - Hábitat, coordinó la realización en 2017 de la Nueva Agenda Urbana Cubana (NAUC), así como de una herramienta para su implementación ${ }^{12}$, sobre la base del documento aprobado a escala global y el Plan de Acción Regional, mediante un proceso de talleres participativos que contó con la colaboración de ONU-HABITAT. A partir de los principios declarados, se elaboró un diagnóstico, se trazaron objetivos y se identificaron líneas de trabajo y acciones para contribuir a mejorar la calidad de vida.

Como resultado del diagnóstico se reconoció el alto grado de urbanización (77.8\%) con densidad de población urbana promedio de 45 hab/Ha, en un sistema de asentamientos planificado y equilibrado, así como el elevado nivel de desarrollo humano, a pesar de la baja renta per cápita, y se prevé un decrecimiento de la población a partir de 2025. Entre las debilidades detectadas se encuentran la estructura institucional vertical, las dificultades para llevar a la práctica los instrumentos de planificación urbana, el poco uso del concepto de edificabilidad y la no

\footnotetext{
${ }^{12}$ El documento está disponible en el sitio web del IPF: www.ipf.gob.cu
} 
percepción de las ciudades como motores de la economía, aun con la propiedad pública del suelo.

Sobre esa base, se elaboró una herramienta que incluye 74 acciones contenidas en 24 líneas de trabajo, entre las que se encuentran planificación, legislación, financiación, economía urbana, vivienda, infraestructura técnica, movilidad, riesgos y cambio climático, a partir de lo cual el Consejo de Ministros aprobó el 26 de diciembre de 2019 el Plan del Estado para la NAUC.

\subsubsection{La Red de Centros Históricos}

La Oficina del Historiador de la Ciudad de La Habana $(\mathrm{OHCH})$, creada en 1938, intensificó desde finales de la década del 70 los trabajos de conservación del principal centro histórico de la ciudad ${ }^{13}$ que, a pesar de su elevado deterioro por décadas de abandono sin mantenimiento, existía gracias a que los cambios políticos, sociales y económicos acaecidos a partir de 1959 abortaron el Plan Piloto desarrollado para La Habana por el urbanista Catalán Jose Luis Sert ${ }^{14}$. Esa labor previa resultó decisiva para la inclusión del centro histórico y el sistema de fortificaciones en la lista del patrimonio mundial en 1983, lo cual, a su vez, impulsó la continuidad de su desarrollo.

Esta experiencia, que fue pionera en América Latina y cuyo sistema de gestión y resultados ha sido ampliamente reconocido ${ }^{15}$ (RODRÍGUEZ ALOMÁ, 2009), difiere de otras similares de la región, fundamentalmente, en cuanto a su enfoque social, ya que la conservación no solo se dirige al patrimonio arquitectónico y urbano, sino al ambiente social y cultural, de

\footnotetext{
${ }^{13}$ Como La Habana toda es considerada una ciudad histórica (Según la tesis de doctorado de Felicia Chateloin, defendida en 2008), La Habana Vieja es sólo uno de sus centros históricos, no el único.

14 Este plan proponía demoler todo el centro histórico, dejando sólo una hilera de manzanas como testigo de lo que había sido la ciudad.

${ }^{15}$ El éxito de la experiencia de La Habana Vieja ha sido avalado por los resultados estadísticos, el reconocimiento de los ciudadanos, la obtención de más de una decena de premios internacionales (Dubai, 2000; UNESCO, 2001; Stockholm, 2001; Valencia, 2002; Toledo, 2003; Medellín, 2005; UN Habitat, 2007; "Reina Sofía", 2007; Nápoles, 2007; entre otros) y la valoración de múltiples expertos, como los convocados por la UNESCO, para un ejercicio de valoración realizado en el 2004, a partir de la solicitud que la Oficina del Historiador de la Ciudad $(\mathrm{OHCH})$ hiciera a la ORCALC, entre ellos, el Dr. Sylvio Mutal y el Arq. Fernando Carrión (RODRíGUEZ ALOMÁ, 2009)
} 
manera que una buena parte de los actuales residentes se mantienen habitando el centro, que constituye, por tanto, una ciudad viva y auténtica, y no una escenografía montada para el turismo (MUTAL, 2006; CARRIÓN, 2006).

Esto se logra gracias al rol desempeñado por las entidades estatales y gubernamentales encargadas de la gestión integral, balanceando costos y beneficios, de manera que una buena parte de los ingresos y las plusvalías urbanas que se obtiene se reinvierte en el propio proceso de conservación del centro histórico, dirigido, fundamentalmente, al subsidio cruzado de los programas sociales, entre los cuales se encuentran, la vivienda, la salud, la educación, la tercera edad y otros grupos poblacionales vulnerables (PÉREZ; IGLESIAS, 2014).

A partir de las experiencias obtenidas en la conservación de La Habana Vieja, en la última década se fueron activando las oficinas del conservador en lugares donde ya existían, y se crearon en otros, ya que todas las ciudades cubanas conservan sus centros históricos sin transformar, aunque muy deteriorados por la falta de mantenimiento. De hecho, son cuatro los centros urbanos de Cuba inscritos en la lista del patrimonio mundial: además de La Habana (1983), Trinidad (1988), Cienfuegos (2005) y Camagüey (2008).

El conjunto de Oficinas del Historiador se ha integrado en una red, coordinada por el historiador de La Habana, con vistas a intercambiar conocimientos, experiencias, aprendizajes y buenas prácticas que contribuyan a promover la conservación del patrimonio material e inmaterial en toda la isla.

\subsubsection{La resiliencia urbana.}

La labor de la Defensa Civil cubana dirigida a la protección de la población y los bienes materiales ante los eventos meteorológicos 
extremos también ha sido ampliamente reconocida a escala internacional, sobre todo, porque se minimiza la pérdida de vidas humanas ${ }^{16}$. Desde el nivel nacional hasta la escala local, existen planes precisos y la población ha sido capacitada en cuanto a cómo actuar, fundamentalmente ante los eventos hidrometeorológicos como inundaciones, penetraciones del mar y fuertes vientos, que son los más frecuentes, y que acompañan a los huracanes.

Esta práctica se ha ido perfeccionando sobre la base del incremento de la solidaridad y colaboración entre los propios pobladores, de manera que las viviendas menos vulnerables en cuanto a localización y estructura sirven de abrigo temporal en estas situaciones a familias vecinas, con lo cual se evita su traslado a albergues en zonas más distantes. Otros programas puestos en práctica durante la última década promueven el concepto de "núcleo refugio"17, de manera que cada familia que habite una vivienda vulnerable ante estos eventos pueda protegerse, junto a sus pertenencias más valiosas, al menos en un espacio más seguro de la propia casa, tal y como ocurrió históricamente en la vivienda vernácula rural, que contaba con el "vara en tierra"18 para tales fines (GONZÁLEZ COURET, 1993).

Por otra parte, la "Tarea Vida", que es un programa estatal dirigido a enfrentar el cambio climático, ha estado promoviendo durante los últimos años, investigaciones y proyectos comunitarios encaminados a transformar los asentamientos costeros, mayoritarios en la isla, en aras de su adaptación, además de prácticas encaminadas a la mitigación de sus efectos (GONZÁLEZ; OLIVERA, 2018).

\footnotetext{
${ }^{16}$ En materia de Defensa Civil y Prevención de Riesgos Cuba ha compartido sus experiencias en el Caribe y el Pacífico Sur (HERNÁNDEZ BARRIOS, 2017). También la OPS y la OMS promueven la experiencia cubana frente a emergencia y desastres (BOLETÍN, 2016).

${ }^{17}$ Se refiere a las viviendas con techos ligeros, en cada una de las cuales debe habilitarse, al menos, un espacio con cubierta pesada, menos vulnerable a los fuertes vientos.

18 Pequeño espacio de sección triangular ubicado al fondo de la vivienda, cuya forma aerodinámica lo hacía invulnerable al viento y, por tanto, apropiado como refugio de la familia en caso de huracanes.
} 


\subsubsection{Las unidades de gestión local del hábitat}

Se trata de una experiencia teórico - práctica, cuyo origen tuvo lugar en varios trabajos desarrollados con vistas a la defensa de tesis de la Maestría en Vivienda Social y el Doctorado en Arquitectura. La investigación y su aplicación, fueron realizadas en la ciudad de Ciego de Ávila, localizada en el centro de la isla, por funcionarios de la Dirección Provincial Inversionista de la Vivienda, que, a su vez, eran alumnos de los programas de maestría y doctorado de la Universidad Tecnológica de La Habana (GONZÁLEZ COURET et al, 2011). La intención era lograr una gestión integrada y descentralizada del proceso inversionista de la vivienda a escala de barrio o Consejo Popular, que es la estructura básica del sistema de gobierno en Cuba, diferente de la tradicional forma de gestión que dispersaba en varias zonas urbanas los recursos disponibles, según las prioridades de cada sector, con lo cual no era posible lograr un impacto integral visible en la elevación de la calidad del hábitat en una determinada zona. Esta experiencia complementa la descentralización de las decisiones, en cuanto a la asignación de recursos, con la generación endógena.

Para ello se realizó una propuesta, científicamente fundamentada y puesta en práctica, de Unidades Locales para la Gestión del Hábitat (ULGH), cuyo impacto positivo fue demostrado (Figura 1). Esta experiencia, conjuntamente con otras que se exponen en el presente artículo, fue reconocida con el Premio Anual de la Academia de Ciencias de Cuba en 2015 (GONZÁLEZ COURET et al, 2015) y en la Bienal de Arquitectura de Costa Rica en 2018. 
Figura 1: Resultados de la acción integral de las Unidades Locales para la Gestión del Hábitat en barrios de la ciudad de Ciego de Ávila.

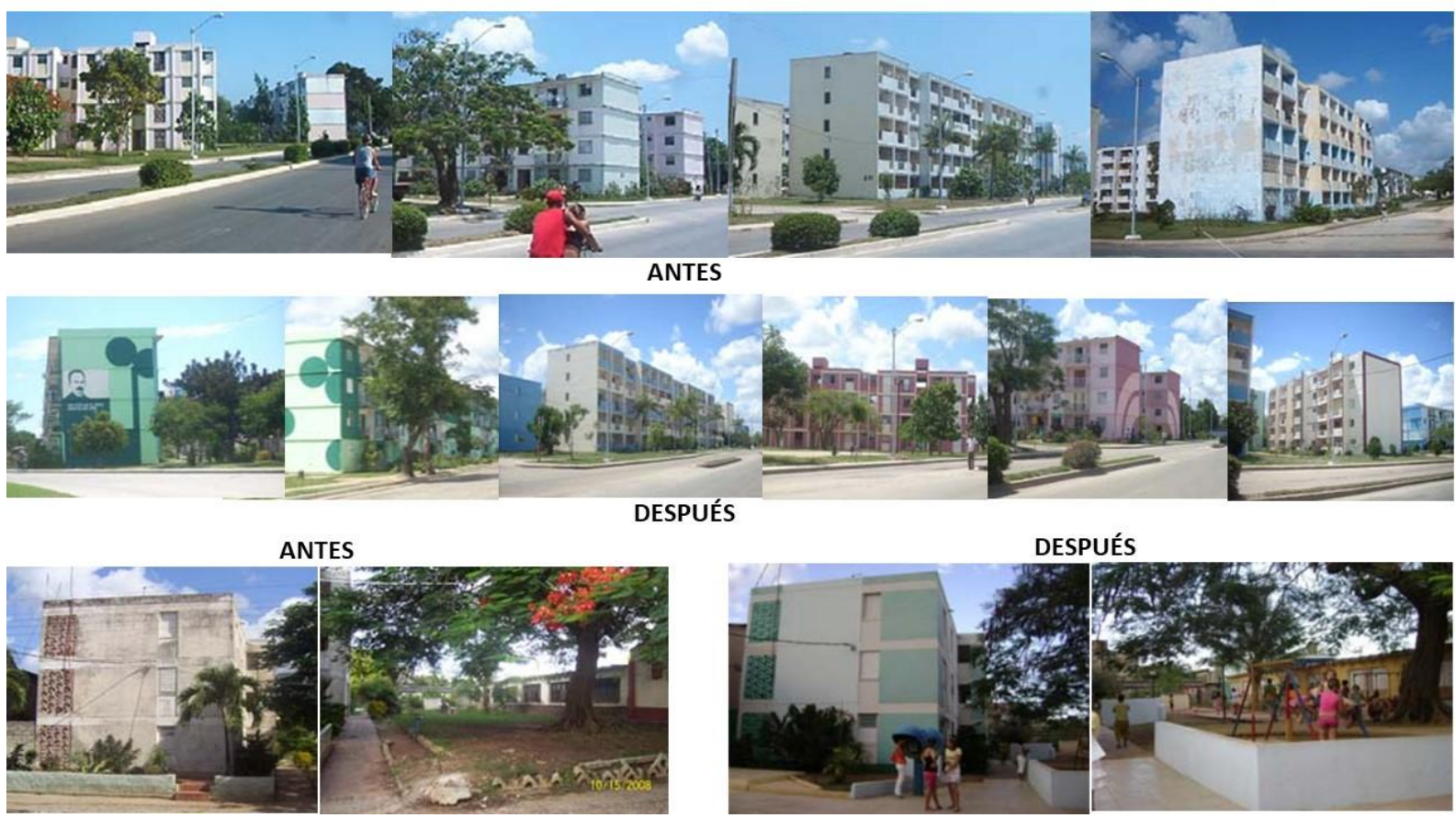

Fuente: Soluciones para mejorar la calidad de la vivienda en Cuba. Trabajo premiado en la XIV Bienal Internacional de Arquitectura, San José de Costa Rica, 2018.

\subsubsection{Los edificios de apartamento en zonas históricas.}

Este resultado parte del convencimiento sobre la necesaria rehabilitación integral de los centros urbanos con vistas a conservar, no solo el patrimonio, sino la población residente, y evitar la extensión de las ciudades. Pero también tuvo como base la evaluación de las soluciones arquitectónicas de los edificios de apartamentos construidos por las microbrigadas en los centros urbanos durante los años 80's, cuyas deficientes condiciones de habitabilidad, de manera general, denotaban la urgencia de recomendaciones y regulaciones normativas que orientaran el diseño de nuevos edificios a ejecutar en estos contextos tradicionales compactos.

Así, se investigó durante varios años la tipología volumétrico espacial de los edificios de apartamentos construidos en diferentes épocas en diversos contextos urbanos consolidados de la ciudad de La Habana, y 
su comportamiento ambiental (térmico y visual), con vistas a proponer recomendaciones de diseño para estos casos, que permitieran resolver la contradicción entre el necesario aprovechamiento del suelo urbano y la garantía de un ambiente interior apropiado, por medios pasivos, que contribuyera a reducir la demanda energética. Ambas condiciones constituyen requerimientos contrapuestos de la sustentabilidad urbana.

Como resultado de los levantamientos, cuestionarios, mediciones, simulaciones automatizadas y propuestas de diseño, se elaboraron recomendaciones en cuanto a indicadores arquitectónicos y urbanos de referencia (GONZÁLEZ COURET, 2016), que pudieron ser incluidos en las normas de vivienda, y fueron divulgados y aplicados mediante el programa de maestría en vivienda social en diferentes ciudades del país.

\subsubsection{La autoconstrucción en los centros históricos}

Esta práctica se vincula con la anterior, a partir de investigaciones desarrolladas para la defensa de tesis de Maestría en Vivienda Social, pero en este caso, en la ciudad de Cienfuegos, donde un maestrante, perteneciente al Programa de Arquitectos de la Comunidad e integrante del Grupo ALBOR, demostró que, aplicando los enfoques teóricos desarrollados anteriormente y manteniendo una estrecha relación arquitecto de la comunidad - cliente, era posible lograr soluciones arquitectónicas de elevada calidad mediante la autoconstrucción en contextos históricos de valor, como el centro de la ciudad de Cienfuegos, incluido en la lista de patrimonio mundial ${ }^{19}$.

Aquí también se demuestra que, aunque la tendencia del gusto popular se aleje de la arquitectura "culta", siguiendo las recetas ofrecidas por albañiles y contratistas, el diseño participativo favorece el mutuo entendimiento en aras de una mayor calidad habitacional, lo cual es posteriormente reconocido por otros clientes potenciales, quienes

\footnotetext{
${ }^{19}$ Tesis de Maestría no publicada: González Baute, Carlos M. Recomendaciones de diseño para la intervención en el hábitat urbano compacto de la ciudad de Cienfuegos, 2013.
} 
prefieren seguir estas nuevas pautas de diseño que optimizan la relación economía - calidad (Figura 2). Este resultado práctico aplicado también forma parte del Premio de la ACC (2015) y la Bienal de Arquitectura de Costa Rica (2018).

Figura 2: Proyectos realizados por el grupo ALBOR y ejecutados por autoconstrucción en el centro histórico de la ciudad de Cienfuegos, siguiendo el enfoque teórico - metodológico desarrollado.
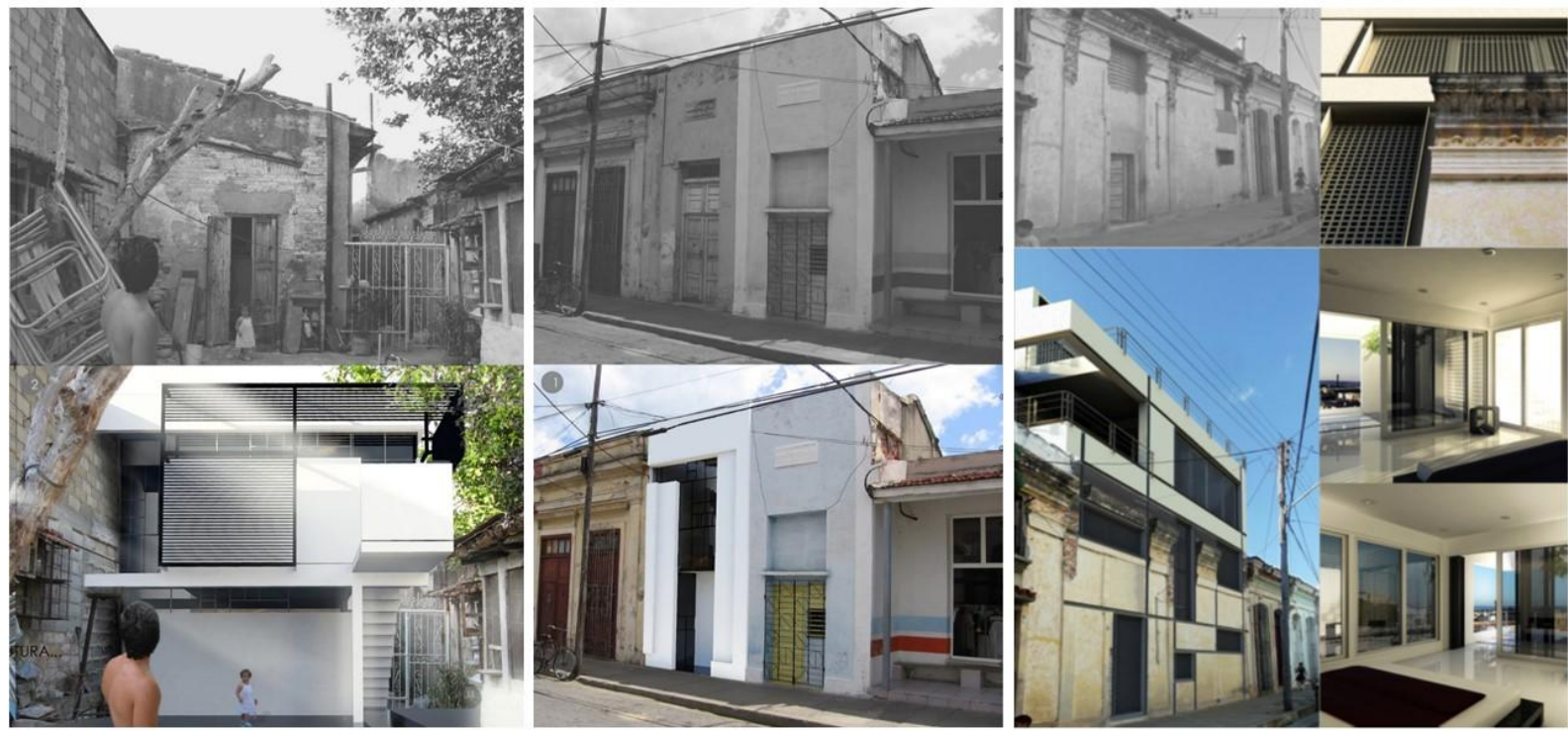

Fuente: Soluciones para mejorar la calidad de la vivienda en Cuba. Trabajo premiado en la XIV Bienal Internacional de Arquitectura, San José de Costa Rica, 2018.

\subsubsection{La vivienda progresiva en zonas urbanas centrales}

En esta ocasión se refiere al resultado de una investigación académica con la cual su autora defendió el Grado Científico de Doctor en Ciencias Técnicas en el Programa de Doctorado en Arquitectura (GELABERT, 2014). Se parte de reconocer que la vivienda es un proceso continuo, ya que el hogar evoluciona, y tanto sus necesidades como las posibilidades económicas van cambiando, por lo cual, el hábitat debe ir transformándose paulatinamente. Sobre esta base, se identifican y evalúan las diversas modalidades de progresividad en cuanto a potencialidades y limitaciones. La vivienda "semilla", predominante en la región para los estratos de menos recursos, restringe el crecimiento en altura, y con ello, el aprovechamiento del suelo y la densidad, a la vez que en etapas 
intermedias de su desarrollo puede afectar la imagen urbana. La modalidad "cáscara", por el contrario, garantiza una imagen acabada desde el inicio, pero resulta más cara que la anterior, mientras el tipo "soporte", más común en países desarrollados y también costosa, requiere una elevada inversión inicial (Figura 3).

Figura 3: Esquema de tipos de vivienda progresiva: semilla, cáscara y soporte.
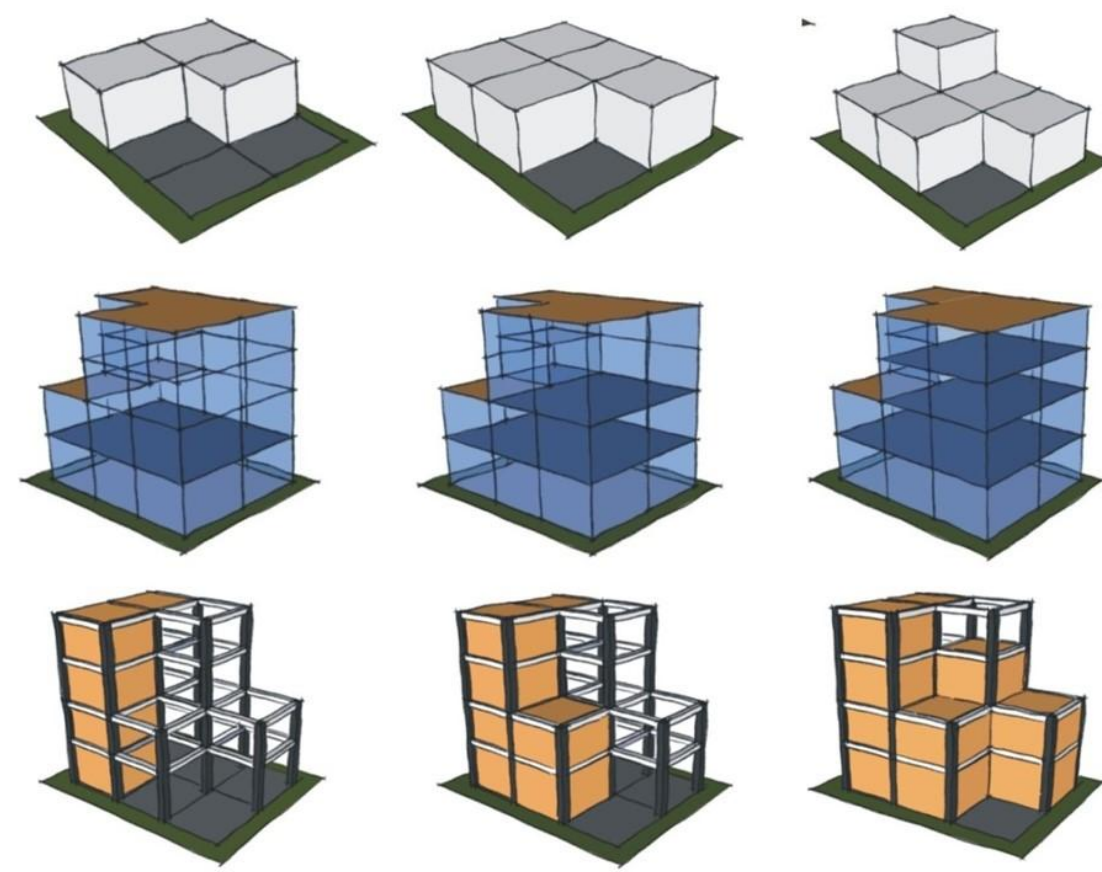

SEMILLA
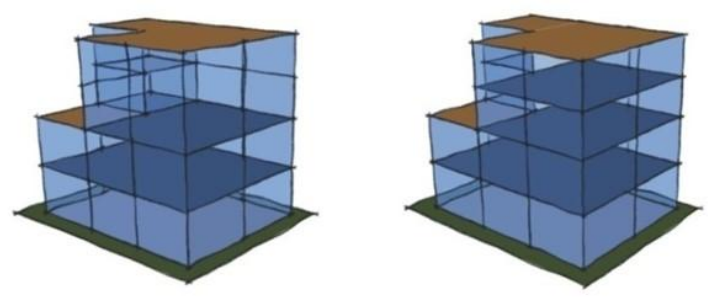

CÁSCARA
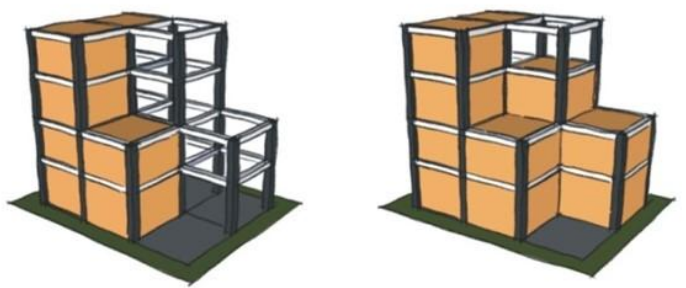

\section{SOPORTE}

Fonte: Gelabert (2014)

Es por ello que, como resultado de la referida investigación, se ha propuesto combinar la potencialidad de las empresas constructoras estatales para ejecutar los soportes con la capacidad de las familias para completar y mejorar progresivamente sus viviendas, según necesidades y posibilidades. La presente fórmula permite reducir los costos, tanto la inversión inicial de la empresa, que no se ve precisada a entregar las viviendas terminadas, como los gastos en los que incurre la familia, que no quedan agravados por la infraestructura. A la vez, se acortan los procesos, al menos el de ocupación inicial de la vivienda, que puede adecuarse a las preferencias de sus habitantes, y es posible garantizar la calidad habitacional, tanto hacia el interior como para el espacio urbano (Figura 4). 
Figura 4: Proyectos de edificios de vivienda progresiva en diferentes contextos urbanos de La Habana, combinando la acción estatal con la autoconstrucción, realizados por los estudiantes (hoy arquitectos) Anelys Lay, Danessa Urquiola e Irasel Ulacia.
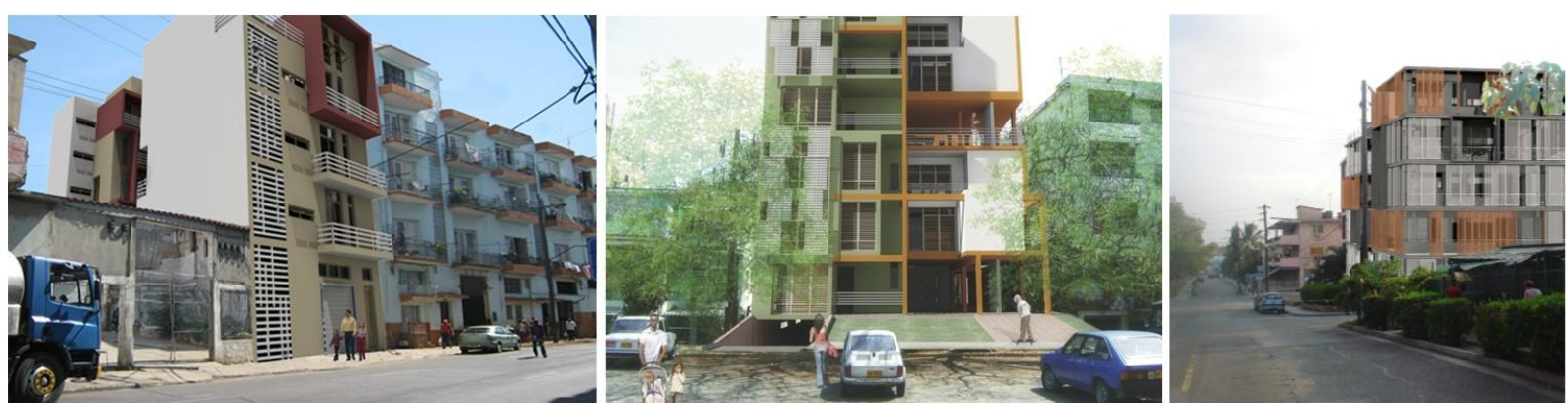

Fuente: Soluciones para mejorar la calidad de la vivienda en Cuba. Trabajo premiado en la XIV Bienal Internacional de Arquitectura, San José de Costa Rica, 2018.

\section{Discusión}

Las experiencias presentadas combinan realizaciones prácticas promovidas por diversas instituciones y propuestas teóricas, parcialmente aplicadas, desarrolladas a partir de investigaciones académicas, en aras de mostrar las potencialidades que existen y que pueden maximizarse cuando los esfuerzos se combinan y complementan. Una particularidad cubana radica en la economía centralmente planificada, en camino hacia un proceso de descentralización parcial que favorecerá la participación y la sustentabilidad de los procesos locales con un carácter endógeno. Por otro lado, a pesar de que no existe actualmente una política integral de vivienda, ni se ha actualizado la ley modificada en 1986, la clara voluntad del Estado para mantener el equilibrio entre la sociedad y el mercado, así como el proteger a los sectores vulnerables continúan siendo una garantía de justicia social.

Con independencia de las diferencias en los enfoques predominantes en diversos momentos, la presencia del sector no estatal es una realidad ya incuestionable e irreversible en la economía y la sociedad cubana. De lo que se trata es de articularlo de la mejor manera posible con el sector estatal, ambos con mayor autonomía de gestión, aunque la intención de 
ralentizar la estratificación social y mantenerla controlada dentro de ciertos límites, siga constituyendo un objetivo esencial. El ámbito del hábitat urbano es uno de los que con mayor urgencia necesita de la integración armónica de ambas formas de gestión económica en aras del progreso social y el aumento de la calidad de vida. En cualquier caso, existen muchos ejemplos que demuestran que también este constituye un sector de la población socialmente comprometido, que dedica parte de sus ganancias a impulsar proyectos comunitarios y de desarrollo local (PÉREZ; IGLESIAS, 2014).

Otra característica de la sociedad cubana contemporánea, que constituye, sin dudas, una fortaleza, es su sentido de comunidad, solidaridad y colaboración, así como su organización social en todos los niveles y esferas ${ }^{20}$, y la poca dependencia del consumo, una vez que ha estado sometida durante décadas a situaciones de escasez y racionamiento de productos básicos para la subsistencia. Estas condiciones han permitido desarrollar, además, un elevado grado de resiliencia, iniciativa y creatividad, imprescindible para la supervivencia en condiciones difíciles $^{27}$. Por otra parte, la prioridad dada a la educación y la salud en las políticas estatales ha permitido alcanzar elevados índices de desarrollo humano y contar con un importante capital científico técnico que se revierte en beneficio de la propia población, además de las probadas posibilidades de colaboración internacional.

La demostrada resiliencia, el bajo compromiso con la sociedad de consumo, el capital humano generado y la favorable relación entre el índice de Desarrollo Humano y la Huella Ecológica (WWF, 2006) son algunos de

\footnotetext{
20 Esto ha quedado fehacientemente demostrado en situaciones de emergencia como fenómenos meteorológicos extremos (huracanes), epidemias (Dengue, Covid-19, entre otras), o crisis económicas como el llamado "Período Especial" en los 90.

21 La capacidad de la sociedad cubana contemporánea para afrontar impactos, recuperarse y continuar desarrollándose (resiliencia) se basa, en gran medida, en la iniciativa y creatividad de la población, gracias a lo cual, por ejemplo, circulan aun vehículos automotores que superaron con creces su vida útil, y funcionan industrias cuyas piezas de repuesto el bloqueo de Estados Unidos ha impedido adquirir. La "Revolución Energética" de 2005 permitió sustituir equipos electrodomésticos que la población, con su ingenio, había podido mantener funcionando por más de medio siglo o habían logrado crear a partir de componentes reusados o reciclados.
} 
los factores que han hecho que expertos internacionales consideren a Cuba como el país ideal para emprender un nuevo camino hacia el desarrollo sustentable (STRÖMDAHL, 2010).

Aunque se desarrollan investigaciones básicas, la vocación principal de la ciencia cubana va dirigida a su aplicación en la búsqueda de solución a los propios problemas de la sociedad, a la vez que se generan nuevos conocimientos. Algunas de las experiencias académicas mostradas se basan en la articulación de las funciones sustantivas de la universidad (Investigación - formación - extensión), aun cuando los resultados no siempre tengan una aplicación directa e inmediata, debido, fundamentalmente, al desconocimiento, por parte de los propios sectores productivos, de las ventajas que proporciona la innovación, o a su resistencia al cambio. La relación universidad - ciencia - producción es muy cercana en ciertas esferas como la medicina, la industria biotecnológica y farmacéutica, pero aún algo más distante, por ejemplo, en la producción de alimentos, las construcciones, la vivienda y el hábitat urbano.

\subsection{Retos}

De las experiencias mostradas y la discusión de sus resultados pueden derivarse algunos de los principales retos que el país debe enfrentar en el campo del hábitat urbano para el futuro mediato. El primero es, sin dudas, la conclusión y aprobación de la Política y la Ley de la vivienda, cuestión que ha venido posponiendo por casi diez años, a partir de los lineamientos asumidos para la política económica y social. Pero para que sean realmente efectivas, ambas (la política y la ley), deben partir de un enfoque integral que abarque la dimensiones ambiental, económica y social, aplicable en el ordenamiento físico, desde la escala territorial hasta el ámbito interior del hogar. Para ello será necesario considerar la evolución que ha experimentado la familia cubana durante las últimas décadas y su diversidad, reconocida en la Constitución de la República recientemente aprobada en 2019. Por otra parte, la intención manifiesta de proteger a los 
sectores vulnerables de la población deberá incluir, no solo a la tercera edad en una sociedad cada vez más envejecida, sino a los jóvenes que constituyen el relevo, para que puedan construir su proyecto de vida.

El carácter integral en la visión del hábitat urbano tendrá que desterrar, cuanto antes, el enfoque sectorial que ve a la vivienda como "un objeto a construir" más que como espacio de vida, producción y reproducción, además de componente básico de las ciudades, que se encuentran entre lo más valioso de la cultura en cualquier sociedad humana. Para ello es casi imprescindible la unificación de la gestión de la vivienda, la ciudad y el territorio bajo una sola entidad que garantice la articulación armónica entre las tres escalas de trabajo y sus correspondientes problemáticas.

Otro importante reto para elevar la calidad del hábitat urbano es el aprovechamiento del capital humano, específicamente, el personal técnico profesional capacitado en los campos de la arquitectura, el urbanismo, la ingeniería y otras esferas afines, para que aún desde el sector no estatal puedan realizar las labores de alto valor agregado para las que han sido capacitados. El marco legal del "trabajo por cuenta propia" y los oficios y profesiones que podrán desarrollarse por este concepto, se encuentra actualmente en revisión, lo cual ofrece una excelente oportunidad para que algunas labores vitales en el campo del hábitat urbano, puedan ser consideradas y autorizadas. No obstante, esta es una condición necesaria, pero no suficiente, ya que también se requiere de una adecuada coordinación armónica entre los diferentes modelos de gestión (estatal, no estatal y cooperativo) que pueden intervenir en la conformación y transformación del ámbito urbano, de manera que cada cual asuma las funciones para las que esté más capacitado y sus roles se complementen. Pero todo eso deberá ser adecuadamente estructurado y legislado en un futuro mediato. 
Por otro lado, será necesario, también, dinamizar la economía local, a partir de un efectivo proceso de descentralización y regulaciones que permitan potenciar las posibles fuentes de financiamiento endógeno, y que aprovechen las fortalezas locales en aras del mejoramiento del hábitat y el ambiente urbano. En todo esto, a su vez, la aplicación de la ciencia juega un rol determinante, mediante la asociación del sector productivo, los gobiernos locales y las propias comunidades con las universidades y centros científicos, a partir de las posibilidades reales de autonomía económica que sirvan de base a la toma de decisiones para la introducción de resultados de investigación, el desarrollo de proyectos innovadores y de experiencias "piloto" demostrativas, cuya aplicación pueda ser posteriormente extendida, de acuerdo con la evaluación en la práctica de sus resultados parciales.

En cuanto a la materialización física del hábitat urbano y su expresión, uno de los principales retos radica en cómo resolver la contradicción entre la necesaria conservación del patrimonio edificado (en tanto elemento esencial de la historia, la cultura y la identidad) y las nuevas demandas de la vida contemporánea y futura, que no siempre se satisfacen con la espacialidad heredada. Esto resulta esencial en una sociedad que, como la cubana, ha podido conservar un patrimonio histórico, altamente valorado, con el cual la calidad arquitectónica y urbana de lo creado durante los últimos más de 60 años, no compite.

Por último, el reto mayor podría ser cómo mantener la inclusión social a escala urbana, que es una demanda global actual, y que ha sido una característica común en el hábitat cubano de la segunda mitad del siglo XX, a pesar de las diferencias heredadas de la sociedad anterior. Algunas transformaciones económicas operadas durante la última década en aras de dinamizar la economía y potenciar los ingresos al país han incrementado la estratificación social (no siempre en relación con el aporte), lo cual afecta el cumplimiento de este objetivo, y, de hecho, sus impactos ya comienzan a apreciarse, al menos en La Habana. Si bien estas 
medidas son anteriores a la última década y ya habían generado diferencias en el acceso a bienes de consumo y algunos servicios, éstas han comenzado a apreciarse en el hábitat, después de la autorización de la compra-venta de casas en 2012.

A partir de la autorización del mercado de viviendas de segunda mano, las diferencias de ingresos y de capital acumulado han comenzado a traducirse en una concentración de la población de mayores recursos, comprando en los lugares más apreciados de la ciudad, ya sea por sus valores históricos, sociales, urbanos, ambientales, culturales o su interés para el turismo (en resumen, la franja norte), y un desplazamiento de la población que en estas zonas urbanas habitaba inmuebles de gran calidad y alto grado de deterioro, cuyos escasos ingresos les impedían mantener.

\section{Conclusiones}

La ciudad informal, surgida como consecuencia del fracaso del modelo urbano moderno asociado a la vivienda social masiva, pasó de ser un problema para convertirse en una oportunidad, que estimuló el cambio de enfoque y el rol del Estado en las corrientes neoliberales, pero no es sustentable, y no debe asociarse el derecho a la ciudad con el de la producción social del hábitat informal.

Las operaciones de rescate de los centros históricos, así como los frentes de aguas o las antiguas zonas industriales mediante operaciones inmobiliarias no deben generar el desplazamiento de la población de menos recursos ni la gentrificación urbana en aras de recuperar plusvalías, lo cual niega los ODS y la nueva agenda urbana.

Las circunstancias condicionantes en Cuba han respondido al ideal de justicia social, y los cambios de enfoque asumidos responden a la contradicción entre la necesidad de eliminar trabas que limitan la solución del problema y el afán de evitar el incremento de la estratificación de una 
sociedad que ha intentado conservar el mayor grado posible de justicia y equidad.

La experiencia de la última década está signada por la aprobación de documentos rectores que trazan las pautas de hacia dónde se pretende encaminar el desarrollo del país en el futuro mediato. No obstante, aún no existe un órgano rector único ni una ley y política de vivienda.

Las experiencias presentadas combinan resultados institucionales oficiales con investigación académica en iniciativas teóricas o parcialmente aplicadas y demuestran que las potencialidades se maximizan cuando los esfuerzos se combinan y complementan.

Las fortalezas del modelo cubano se resumen en el sentido de comunidad, solidaridad, colaboración, organización social, resiliencia, iniciativa, creatividad, desarrollo humano y capital científico técnico, así como la presencia de una economía centralmente planificada, que debe ser complementada con una descentralización que estimule la generación endógena y el desarrollo local.

Entre los retos futuros se encuentran la necesaria aprobación de la ley y la política del hábitat urbano con enfoque integral, bajo el liderazgo de una única entidad; la articulación entre las diversas modalidades de gestión en aras de mantener la justicia y equidad, evitando la estratificación urbana, así como el logro de una mayor colaboración entre la universidad y sector productivo en este campo, aprovechando el capital científico - técnico, para combinar la conservación del patrimonio con las nuevas demandas de la vida contemporánea.

\section{Referencias}

ALONSO ECHEVERRÍA, I. El modelo de vivienda post-covid; resiliente, colaborativo y ecológico. En: El País, 20 junio 2020. Disponible en: 
https://elpais.com/elpais/2020/06/22/alterconsumismo/1592811834_935369.h tml. Accedido en: 12 jun. 2021.

BENÉVOLO, L. Historia de la Arquitectura Moderna. Tomo I. La Habana: Edición Revolucionaria, 1975.

BJUR, Hans; GAVATIN, Margareta, Research and Development in Spatial Planning and Urban Management. En: Swedish Planning Towards Sustainable Development. Gavle: Westlund and Soner , 1997, p.149-154.

BOLETIN (Boletin de la Cooperación Técnica). OPS/OMS. Diciembre 2016. Disponible en: www.paho.org/cub/index.php?option=com_content\&view=article\&id=665:0 ps-oms-promueve-experiencia-cubana-frente-a-emergencias-y-desastres\& Itemid=528. Accedido en 12 jun. 2021.

CARRIÓN, Fernando. El futuro está en el ayer: La Habana Vieja, una plataforma de innovación. En: Una experiencia singular. Valoraciones sobre el modelo de gestión integral de La Habana Vieja, Patrimonio de la Humanidad. Editado por UNESCO / Oficina del Historiador de la Ciudad de La Habana. La Habana: Editorial Boloña, 2006, p. 173-198.

CEPAL (Comisión Económica para América Latina y el Caribe). Panorama Social de América Latina. Santiago: CEPAL, 2019.

DÍAZ-CANEL BERMÚDEZ, Miguel; NÚÑEZ JOVER, Jorge. Gestión gubernamental y ciencia cubana en el enfrentamiento a la COVID-19. Anales de la Academia de Ciencias de Cuba, vol. 10, n.2, 2020. Disponible en: http://www.revistaccuba.cu/index.php/revacc/article/download/881/887 . Accedido en: 12 jun. 2021.

DOCUMENTOS (Documentos del 7mo. Congreso del Partido aprobados por el III Pleno del Comité Central del PCC el 18 de mayo de 2017 y respaldados por la Asamblea Nacional del Poder Popular el 1 de junio de 2017). Disponibles en:

\section{http://www.granma.cu/file/pdf/gaceta/\%C3\%BAltimo\%20PDF\%2032}

Accedido en: 1 jun. 2020. 
FRANCO PARELLADA, Xiomara. Sistema de asentamientos poblacionales: problemas de la franja inferior. Planificación Física-Cuba. s.I., n. 2, p. 22-30, 1989.

GELABERT, D. Vivienda Progresiva como solución alternativa para la ciudad de La Habana. Sevilla: Unidad Internacional de Andalucía, 2014. GONZÁlEZ COURET, Dania. Hábitat rural. Pasado, presente y futuro. Arquitectura y Urbanismo. vol. 14, n.2, p. 51-59, 1993.

GONZÁlEZ COURET, Dania; ÁlVAREZ, Norberto; ÁGUILA, Odalia; PÉREZ, Deremis. Unidades locales para la gestión integral del hábitat. Experiencia Cubana. Revista INVI, n. 76, p. 167 - 198, 2011.

GONZÁLEZ COURET, Dania et al. Soluciones para mejorar la calidad de la vivienda en Cuba. Anales de la Academia de Ciencias de Cuba, vol. 5, n. 3, 2015.

GONZÁLEZ COURET, Dania. Edificios de apartamentos de altura media en La Habana. Evaluación. Cuadernos de vivienda y urbanismo. 10, p. 2 - 15, 2016. Doi:10.11144/Javeriana.cvu10-20eaam

GONZÁLEZ COURET. Dania. El regreso a la ciudad tradicional. Vivienda social de los 80's en La Habana. Revista INVI, vol. 35, n. 98, p: 45-74. 2019. doi:1010.4067/S0718-835820

GONZÁLEZ COURET, Dania. La industrialización de la vivienda en Cuba. Década del 70. Arquitectura y Urbanismo. vol. 42, n.1, p. 34-47, enero - abril 2021.

Disponible

en:

https://rau.cujae.edu.cu/index.php/revistaau/article/view/604/569 . Accedido en: 12 jun. 2021.

GONZÁLEZ, Gonzalo; OLIVERA, Andrés. Adaptación al cambio climático en la recuperación del huracán Irma. En: Memorias del Congreso Internacional Medio Ambiente Construido y Desarrollo Sustentable. CCIA. La Habana: CUJAE, 2018.

GUDAY, Olga. Ciudades después de la pandemia: así cambiarán. Huellas by Sareb, 28 oct. 2020. Disponible en: www.huellasbysareb.es/en-primera-persona/ciudades-despues-covid/ Accedido en: 12 jun. 2021. 
HABITAT 1976. Report of habitat: United Nations Conference on Human Settlements, Vancouver 31 Mayo - 11 Junio 1976, Nueva York, 1976.

HERNÁNDEZ BARRIOS, Manuel Alejandro. La Defensa Civil Cubana un sistema fuerte, enérgico y previsor. Mesa Redonda. [online]. 20 jun. 2017. Disponible en: mesaredonda.cubadebate.cu/mesa-redonda/2017/07/21/la-defensa-civil-cub ana-un-sistema-fuerte-energico-y-previsor-video. Accedido en: 12 jun. 2021. HIC-AL. Coalición Internacional del Hábitat. Hábitat I, Hábitat II, Hábitat III. México : HIC-AL, 2016.2 Disponible en: http://hic-gs.org/content/HIC_Habitat\%201976\%202016\%20ES.pdf. Accedido en 12 jun. 2021.

MACHORRO, Juan Carlos. Modifica COVID-19 al concepto de ciudades inteligentes. Expok - Comunicación de Sustentabilidad y RSE, 2 junio 2020.

Disponible

en:

https://www.expoknews.com/modifica-covid-19-al-concepto-de-ciudades-i nteligentes/ Accedido en: 12 jun. 2021.

MICONS. Las construcciones 1959 - 1989. Informe a la Asamblea Nacional del Poder Popular. La Habana: Ministerio de la Construcción, 1989.

MUTAL, Sylvio. Valoraciones de los expertos de la UNESCO. En: Una experiencia singular. Valoraciones sobre el modelo de gestión integral de La Habana Vieja, Patrimonio de la Humanidad. Capítulo III. La Habana: Editorial Boloña, 2006, p. 125.

NAVARRETE, David. Turismo gentrificador en ciudades patrimoniales. Exclusión y transformaciones urbano-arquitectónicas del patrimonio en Guanajuato, México. Revista INVI, vol. 32, n. 89, p. 61-84, 2017. doi:10.4067/S0718-83582017000100061

ONU-Hábitat. Versión ejecutiva del Perfil de la Vivienda en Cuba. La Habana, 2014.

ORRSKOG, Lars, Planning After Rio. Some Remarks from a North - Western Corner of the World. En: Architecture as Politics. Stockholm: Royal Institute of Technology, 2002, p. 27-32 
ORTIZ FLORES, Enrique. Notas sobre la producción social de vivienda. Elementos básicos para su conceptualización e impulso. México, D.F: Casa y Ciudad, 1998.

PÉREZ, Martha Oneida; IGLESIAS, Maidolys. Patrimonio y ciudadanía. Experiencias de participación en La Habana Vieja. La Habana: Ediciones Boloña, 2014.

PORTNEY, Kent E. Taking Sustainable Cities Seriously. Economic Development, the Environment and Quality of Life in American Cities. Second Edition. Massachusetts Institute of Technology, 2013.

RED- HÁBITAT. Por el derecho humano a la vivienda. La Paz: Taller de Proyectos e Investigación del Hábitat urbano-rural, 2008.

RED HÁBITAT. Carta Mundial por el Derecho a la Ciudad. En: 1er Concurso de Ensayos sobre el Derecho a la Ciudad, pp. 131 - 136. La Paz: Taller de Proyectos e Investigación del Hábitat urbano-rural, 2009.

RIGOL, Isabel. La recuperación del patrimonio documental en Cuba desde 1959. En: Conservación patrimonial: teoría y crítica. La Habana: Editorial UH, 2012, p. 111-130.

RODRÍGUEZ ALOMÁ, Patricia. Gestión del desarrollo integral de los centros históricos. Tesis en opción al grado científico de Doctor en Ciencias Técnicas. Universidad Tecnológica de La Habana, 2009.

ROJAS, Ángela. Planeamiento, flexibilidad, valores. En: Conservación patrimonial. Teoría y crítica, pp. 53-70. La Habana: Editorial UH, 2012.

SEGRE, Roberto. La vivienda en Cuba: República y Revolución. La Habana: Departamento de Actividades Culturales de la Universidad de La Habana, 1985.

SEGRE, Roberto. Arquitectura y urbanismo de la Revolución cubana. La Habana: Editorial Pueblo y Educación, 1989.

STRÖMDAHL, J. Kubas omställning till ekologisk hållbarhet. Stockholm: Langholmen, 2010.

Disponible en: www.adlibris.com/se/bok/kubas-omstallning-till-ekologisk-hallbarhet-97891 74370645. Accedido en: 12 jun. 2021 
TEZANOS, J. F. Tendencias en desigualdad y exclusión social. Madrid: Editorial Sistema, 1999.

THE HABITAT AGENDA. Agenda. Goals and Principles, Commitmenta and Global Plan of Actions. United Nations Conference on Human Settlements (Habitat II). Istanbul, Turkey, 3 - 14 Junio 1996.

TURNER, John. F. Housing by People. Towards autonomy in building environments. London: MarionBoyards. (V. e. usuario, Trad.) Barcelona: Blume, 1976.

VEJAR, Carlos. Y el perro ladra y la luna enfría. Fernando Salinas: Diseño, ambiente y esperanza. México: UNAM, 1994.

WWF (World Wildlife Fund). Informe Planeta Vivo, 2006. WWF, 2006.

Disponible en: https://wwf.panda.org/wwf_news/?109443/Informe-Planeta-Vivo-2006. Accedido en 12 jun. 2021. 